\title{
Exploring the core of crew resource management course: speak up or stay silent
}

\author{
Roar Espevik, Evelyn Rose Saus, Olav Kjellvold Olsen
}

Royal Norwegian Naval Academy, Laksevåg, Norway

\begin{abstract}
The Norwegian Costal Express travels 24/7 along a coast considered as one of the most dangerous littoral areas of the world. It is crucial for safe voyage to speak up when one of the crewmembers discovers a discrepancy or vital new information to the passage that needs to be shared and acted upon. Crew resource management courses are intended to increase safety and we suggest that the key is to enhance the ability to speak up. Watch keepers valued a 4-h course intended to enhance the ability to speak up and improve listening skills as highly relevant (89\%) and educational (69\%). These high scores indicate that this type of training is necessary to improve safety.
\end{abstract}

(Int Marit Health 2017; 68, 2: 126-132)

Key words: teamwork, communication, shared mental models, crew/bridge resource management

"Offshore the hurricane sparked dramatic rescue operations. Several ships ran aground, fishing vessels were wrecked in the beaches and shores. Aircraft and boat routes were cancelled, and people were asked to stay indoors. About the Norwegian Coastal Express nothing was said for it sailed as nothing happened, en route" [1].

\section{INTRODUCTION}

Seafaring is by many reported to be among the world's most dangerous occupations. In one study, Hansen [2] showed that fatal injuries for Danish seamen were 11.5 times higher compared to the workforce ashore. The International Maritime Organisation (IMO) stated shipping as one of the most dangerous industries in the world [3]. A vessel at sea often operates far from hospitals and outside help. Personnel on board must cope with complex and dangerous machinery in a limited space, often made worse by heavy sea and challenging navigation. Serious consequences of wrongdoings such as collisions and explosions are present, with a potential to be enormous and tragic. To prepare for such a high-risk environment and to avoid accidents, training becomes a major concern for maritime organisations.

After several major accidents in the 7os, the airline industry acknowledged that technical competence was not sufficient to guarantee safe performance [4]. This led to the development of crew resource management (CRM) training. Thirty years later the CRM training has been transferred to other high risk-organisations, such as healthcare, military, nuclear plants and maritime organisations.

The most widely used team training in high-risk organisations today is connected to the CRM concepts and billions of dollars are spent on training each year. Salas et al. [5] offers a broad definition: "family of instructional strategies that seeks to improve teamwork by applying well tested training tools targeted at specific content (i.e. teamwork knowledge, skills, and attitudes)" [5].

Hence, when transferred from aviation to the maritime domain there are at least three challenges connected to CRM training. Firstly, with such a broad definition there are different opinions and some disagreement to what CRM training consists of and how it ought to be carried out (i.e. what to train, how to train it) diffuse the picture. Therefore, and maybe most importantly, there is no certainty of the desired effect (i.e. fewer accidents; [6]). Thirdly, transferring a training strategy from the airline industry, with promising results to the maritime domain, does not necessarily give similar effect. Musson and Heimlich [7], pioneers in the field, state that CRM training tends to be domain-, organisation- and culture-specific and straightforward transfer from one work setting to another has often been ineffective. 
Communication problems are consistently identified as leading cause of system breakdown in high-risk organisations [8]. Safe operations are characterised by a mutual understanding that all team members will state their observations, opinions and recommendations, and actively solicit and consider input from others. Hence, there are strong arguments for a claim that the starting point and core of the CRM training is to make all team members speak up when they have vital information. If no one communicates regardless of reason, other vital CRM concepts essential to successful teamwork such as mutual monitoring, supporting behaviour, shared mental models do not even come into play. The present paper describes a 4-h CRM training programme aimed at enhancing team's ability to speak up as well as their listening skills. The participants' evaluation of relevance, as well as perceived learning effects of the training programme will be studied. The paper is aimed to explore probable differential evaluation of relevance and perceived learning effects between two groups with different experience and position in the hierarchy (i.e. officer of the watch vs. his assistance).

\section{THEORY}

\section{CREW RESOURCE MANAGEMENT AND SPEAK UP BEHAVIOUR}

As suggested, one major challenge is that there is no standard set of competencies, and a wide range of concepts have been defined as CRM [9]. Typical topics seem to be teamwork, shared mental models, leadership, situation awareness, communication and personal limitations [9]. However, at the core of this, there seems to be a common understanding that failure to speak up when situated lower in the team hierarchy often is associated with an increased risk of accidents, both in aviation [10] and health care [11]. Highly reliable organisations manage the tendency towards accidents through collective identifying and managing continuously evolving threats. That is, all team members are charged to scan continuously for threats, and more important, for speaking up when they identify potential threats, regardless of their status in the hierarchy or their defined role [12]. On a ship that is to speak up against the captain with the overall responsibility [13], or the officer of the watch responsible for a safe and secure voyage.

In contexts where errors are few, but at enormous costs, team members are bound to handle every small incident as a learning event. To create such a climate for learning, team members must not only be willing to speak up, but also trust that what they say or do will not be held against them. We claim that this is the core CRM object. Hence, with little willingness to speak up within the team there is no room for other suggested CRM concepts. Thus, we advocate that the starting point for a team is to be aware of the barriers for speaking up and train how to enhance its ability to accomplish this. However, the literature suggests little as to how this is perceived by professionals (e.g. watch keepers on board ships) within the maritime domain.

Røttger et al. [14] studied German navy personnel and found that negative attitudes from senior officers are correlated with bad performance. When 324 bridge officers were asked if they were reluctant to question pilot decisions, $81 \%$ responded "sometimes" and $12 \%$ answered "always". The Canadian Transportation and safety board reviewed 273 incidents from 1987 to 1992 with vessels in Canadian pilotage waters [15]. Hence, there are indications that training to speak up also is important within the maritime domain. Further, such training has the potential to enhance the quality of teamwork and reduce accidents. Burke et.al. [16] state that promoting assertiveness in aviation and health care has been a major challenge due to the resistance from the senior personnel. Studies has shown that second pilots and nurses valued CRM communication training more positive than their counterparts higher in the hierarchy (i.e. surgeon and captain in the cockpit) $[17,18]$. In addition, crews on board navy ships are organised hierarchically, from master and downwards. We suggest that power differences are similar within the maritime domain. Thus, the need for speak up training may be perceived different depending on where you are in the ship's hierarchy.

Despite decades of CRM, training teams in high-risk organisations such as shipping, health care and military organisations, there is considerable uncertainty whether this type of training actually increases safety. CRM courses are intended to increase awareness and skills around the importance of clarity of roles, clear communication, as well as situational awareness. We suggest that the key is to increase safety by getting everyone in the team to share information more easily. However, training to enhance the ability to speak up has to be perceived relevant and learned by the professional workers. Research on CRM has mainly been simulation studies where the subjects studied remarkably often are male undergraduate students or aviators [19]. As Salas et al. [20] put in 2008; "We need to study teams in the wild."

Trainees seem to react positively to training. However, in the studies where the effect was transferred to the job, it was based on a particular scenario or the experience of the trainees (i.e. they were not professionals) [6].

\section{HOW TO TRAIN}

Salas et al. [21] tried to help the transmission of CRM training from aviation to the medical domain by proposing eight critical principles when creating a training programme. 
They underline the importance of identifying key teamwork components (e.g. assertiveness), and to ensure that shared understanding and adaptive behaviours are facilitated. Furthermore, they claimed that team training must promote attitudes and behaviours that are indicative of a learning climate. Finally, ensuring that all team members engaged in closed loop communication, there are opportunities for practice with feedback. Hence, a training programme for the maritime domain must follow the same line of thinking.

\section{RELEVANCE AND LEARNING}

Kirkpatrick's [22] hierarchy is an often-used framework for guiding training evaluation, and consists of four different levels: reactions, learning, behaviour and organisational impact [9]. Reactions cover to what degree the participants find the time spent worthwhile or simply whether they like the training. The learning means that the training was understood and absorbed. Thus, investigating perceived relevance and perceived learning constitute the two basic levels.

Learning consists of acquiring knowledge; and personal knowledge is defined as 'the cognitive resources which a person brings to a situation that enable them to think and perform' [23]. Students' overall perception of learning has been used as an indicator of learning in educational research [24].

\section{HYPOTHESIS}

The Norwegian coast is considered as one of the most dangerous littoral areas of the world. Safe voyage is heavily dependent on seafarers' knowledge, insight, skill and most importantly, their ability to cooperate. On the Norwegian Coastal Express, which travels these waters 24/7, two officers on watch secure a safe passage, the watch leader and his assistant. It is crucial for them to speak up when one of them discovers a discrepancy or vital new information on the passage that needs to be shared and acted upon. Thus, as a team in a high-risk environment, it seems highly appropriate to investigate speak up training. If the watch keepers perceive it as relevant and that actual learning has taken place, it may suggest its importance in the maritime domain. We suspect that bridge teams from the Norwegian Coastal Express, ("the wild") will respond positively to training aimed at making team members speak up to each other. Our suspicion increased when the management of the coastal express ashore voiced opinions on a very strict hierarchy on board.

Thus - H1: maritime teams will report increased perceived learning effects and perceived relevance after training. Different positions in hierarchy may give different result. Gore et al. [17] and Carney et al. [18] reported that surgeons and captains in the cockpit did not see the need for CRM training compared to team members further down the hierarchical ladder.
Table 1. Demographic characteristics of participants $(n=39)$

\begin{tabular}{lc}
\hline Category - Age & $\mathbf{N}(\%)$ \\
\hline 25 years old and younger & $1(2.56 \%)$ \\
$26-30$ years old & $10(25.64 \%)$ \\
$31-35$ years old & $8(20.51 \%)$ \\
$36-40$ years old & $5(12.82 \%)$ \\
$41-45$ years old & $5(12.82 \%)$ \\
$46-50$ years old & $3(7.69 \%)$ \\
$51-55$ years old & $3(7.69 \%)$ \\
$56-60$ years old & $3(7.69 \%)$ \\
$61-65$ years old & $1(2.56 \%)$ \\
\hline
\end{tabular}

Thus - H2: watch leaders will report less perceived learning effects and relevance compared to their assistants.

\section{METHOD/EMPIRICAL SECTION}

\section{PARTICIPANTS}

Forty-three navigators participated in the study, but four persons did not evaluate the training programme. In order to protect the participants' anonymity, they did not report sex. Further analysis is based on 39 participants (Table 1).

One person was 25 years old or younger $(2.56 \%)$, 10 persons between 26 and 30 years old $(25.64 \%)$, 8 persons were between 31 and 35 years old $(20.51 \%)$, 5 persons were between 36 and 40 years old $(12.82 \%)$, 5 persons between 41 and 45 years old (12.82\%), 3 persons between 46 and 50 years old (7.69\%), 3 persons between 51 and 55 years old (7.69\%), 3 persons between 56 and 60 years old ( $7.69 \%$ ) and 1 person between 61 and 65 years old (2.56\%).

\section{PROCEDURE, APPARATUS AND QUESTIONNAIRES}

In a 4-week period, ships from the Norwegian Coastal Express Company, on a regular 8-h stop in the city of Bergen, sent their four navigators to the facility of the Royal Norwegian Naval Academy. The captain and the chief officer are watch leaders while the navigation and security officer function as their assistants. Those four from the same crew then went through the 4-h training together.

\section{PLENARY SESSION}

The first hour the 4 participants were introduced to the importance of and possible barriers to speak up behaviour. Involvement by all participants was enforced through direct questions and presentations of maritime examples and situations where speak up behaviours had impact on the outcome. The goal was to reach a common awareness and understanding within the bridge team, how speak up 
behaviours inflict bridge operations and safe navigation. The next step was to put this insight and common understanding to a challenge aimed to enhance learning. That is, to put the participants in situations where they, as team members, were forced to experience speak up and listening behaviour, and through practice, feedback, reflection and discussion within the group experience and understand the importance and how to overcome any barriers.

\section{GROUP SESSIONS}

Two group sessions were performed focusing on creating shared intentions in teams, thus focusing mutual monitoring to enable more speak up communications and listening skills to create shared mental models within the team (i.e. push vs. pull communication; ref. information).

The first group session lasted $45 \mathrm{~min}$ and was performed in a group of four, both bridge teams together. The group was put in a situation with time constraints where they were blindfolded and, as one team, should solve an unfamiliar problem. After a preset time of $10 \mathrm{~min}$, one of assistants (the youngest) secretly got vital information that if the other team members responded correctly, this would make the team able to solve the problem. This focused on the benefit of push of information (speak up), trust and the shaping, establishing and support of shared mental models through the big five behaviours suggested by Salas et al. [25] as essential CRM teamwork processes. When the task was completed, a subject matter expert facilitated reflection of how the team solved the task. This was intended to enforce the understanding from the plenary session, preparing for the second group session.

The second group session was performed in a simulator where the four matched up in their regular team of two. Simultaneously, the two bridge teams (captain and security officer vs. chief officer and navigation officer) from each crew - a watch leader and assistant - operated a coastal express for $40 \mathrm{~min}$. Both teams sailed a difficult but known part of the Norwegian coast, with challenging naval traffic and weather conditions. A safe voyage depended on communication between the watch leader and his assistant.

When the task was completed, a subject-matter expert facilitated a 40-min reflection of how the two different teams communicated based on the introduction and the former group session. This was intended to enforce understanding and practice.

Upon finishing the 4-h CRM-training, the participants were asked to subjectively evaluate the perceived relevance as well as rate the perceived learning effect. In order to prevent acquiescence, the subjects were told that participation was voluntary and no individual data would be presented to their colleagues or superiors in the company. The questionnaire contained the three following questions measured by a 10 cm Visual Analog Scale (scored 0 - "very little" to 5 - "very much", with steps of one marked on the scale): "To what extent did the training contribute to increased awareness of team processes?"; "What are your perceived learning effects of the training?", "What is your overall impression of the training?"

The first question focusing on awareness of team-processes can reflect perceived relevance since the purpose of the CRM-course was to enhance speak up behaviour to build high performance teams. The second question concerning learning outcome is an evaluation of the participants' perceived learning effect. Overall perception of learning, represented by this item has previously been used as an indicator of learning in educational research [24]. The third question can be an overall evaluation of both the relevance and the perceived learning effects. It also reflects the participants' evaluation of the CRM-training.

The original scores of all the three questions were categorised as low (0-1.9), medium (2.0-3.9) and high (4.0-5.0).

\section{STATISTICAL ANALYSIS}

Descriptive statistics separated for all items of the evaluation questionnaire are presented. Kolmogorov-Smirnov test of normality showed that the distribution was significantly different from a normal distribution for all three (all d's $>0.411, p<0.01$ ), with a skewness towards a high end of the scoring range (skewness between -0.611 to -2.726 ). Because of the violation of normal distribution, non-parametric tests were used. Mann-Whitney U-tests were used to investigate differences between the two categories of bridge team officers based on responsibility (captain/chief officer vs. security officer/navigation officer) regarding the evaluation of all three questions.

\section{RESULTS}

Descriptive statistics for the three questions are presented in Figures 1-3.

Figure 1 shows the frequency of scoring on the item of increased awareness of team processes (low $=0$, medium $=4$, high $=35$ ): $89.74 \%$ of the participants rated the training as high with regard to awareness of team processes, $10.26 \%$ rated the training in the middle category, and no one rated it in the low category.

Figure 2 shows the frequency of scoring on the item of perceived learning effects (low $=0$, medium $=14$, high $=25$ ): $64.10 \%$ of the participants rated the training as high with regard to perceived learning outcome, 35.90\% rated the training in the middle category and no one rated it in the low category.

Figure 3 shows the frequency of scoring on the item of overall evaluation of the whole CRM-training (low $=0$, medium $=4$, high $=35$ ): $89.74 \%$ of the participants rated 


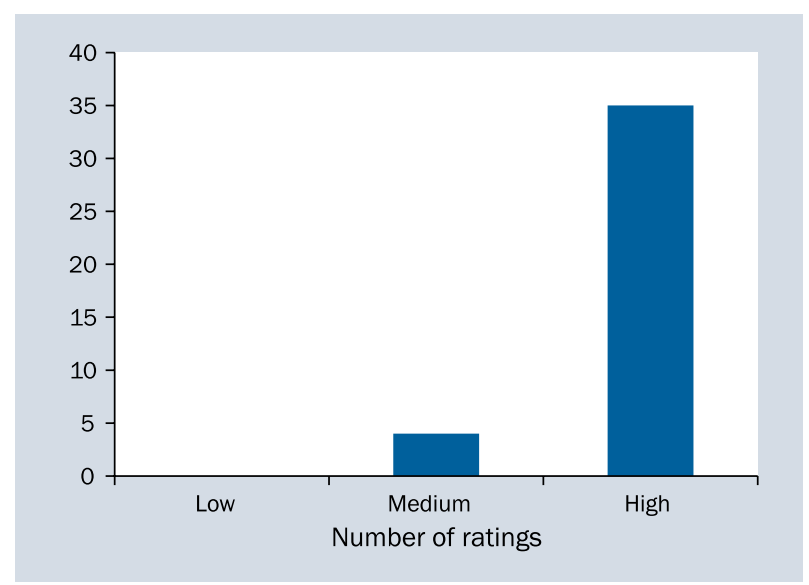

Figure 1. Descriptive statistics for the question evaluating the increased awareness of team-processes from the crew resource management training

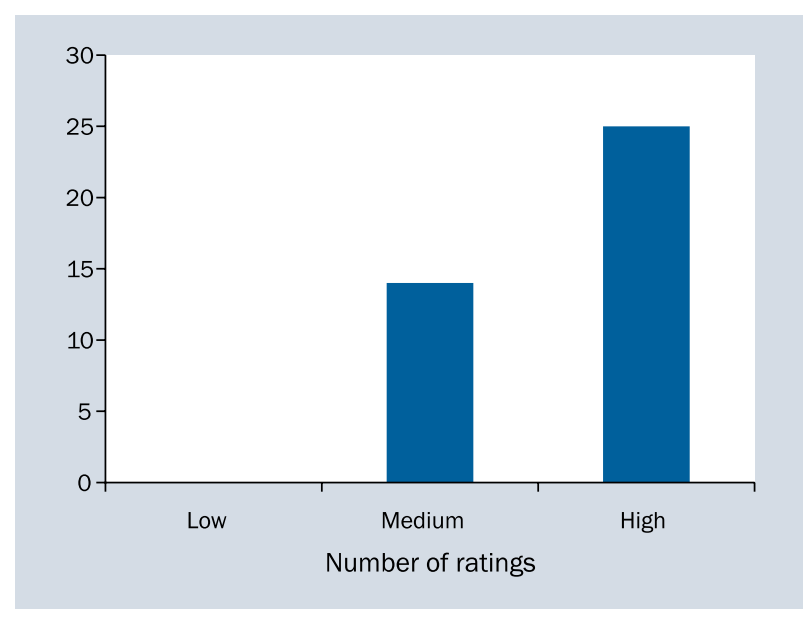

Figure 2. Descriptive statistics for the question evaluating the perceived learning effects from the crew resource management training

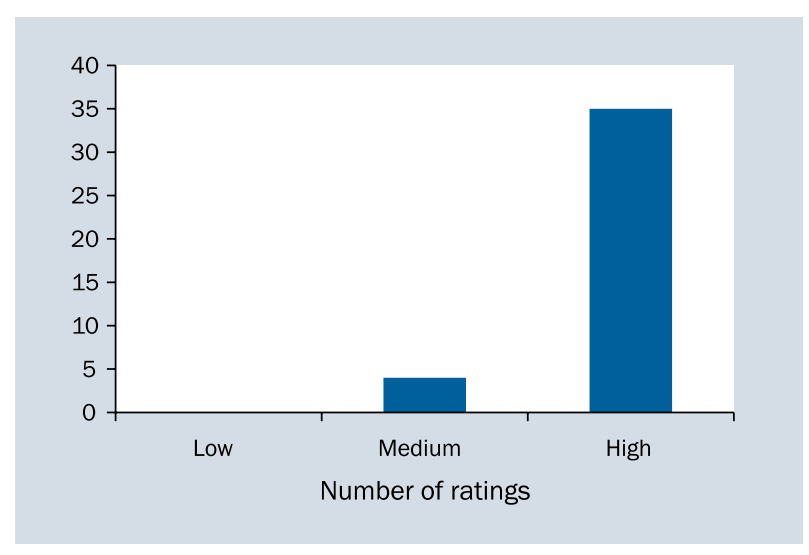

Figure 3. Descriptive statistics for the question evaluating the overall evaluation of the whole crew resource management training the overall evaluation of the CRM-training as high, 10.26\% rated the training in the middle category and no one rated it in the low category.

\section{GROUP DIFFERENCES}

We did not find any group differences, when it comes to team members and their responsibility.

\section{DISCUSSION}

The results revealed high scores on all items in the evaluation of the training programme. Naval officers scoring high on the outcome variables varied from $89.74 \%$ on the question related to relevance and the course as a whole to $64 \%$ on learning. These high scores indicate that this type of training which emphasized speak up behaviour was welcomed by the professionals. Since the programme focused on participants' involvement, this finding was also in line with Birzer [26], who claimed that student involvement is a crucial element in order to increase effects of training. When using Kirkpatricks [22] levels for evaluation of training, the participants positive responses indicate that the training received scored high on level 1 , reactions (i.e. did the participants think the training was worthwhile?) and level 2, learning (i.e. the principles, facts and skills where understood and absorbed by the participants). It is also in line with recommendations from Salas et al. [6], who in a review of training within high-risk organisations concluded that training that concentrates on practice, reflection and a cognitive approach received high scores on reactions and learning.

We designed the training programme based on 8 principles [21]. Issenberg et al. [27] presented a review article that identified 10 features that lead to effective learning in medical simulations. The features included the possibility of a controlled environment, feedback, clearly defined learning outcomes, and that simulation permits individualised learning. Weller [28] reports that medical students set great store by simulation-based learning, and especially the opportunity to apply theoretical knowledge in a safe and realistic setting. All of these features were present in the training programme and could account for the high evaluation presented. Hence the findings in the present study broaden the field regarding effective application of CRM speak up training and applicable also in the maritime domain. It should therefore be integrated into future literature reviews regarding CRM training for bridge teams.

Although high scores on the evaluation of the training programme were obtained independently of experience and position, it came as a surprise that there were no differences between watch leaders and assistants. This may indicate that the power distances on board are lower than anticipated with a flatter hierarchy that may in itself lower the 
barriers to question the masters' decisions. It also suggests that speak up behaviour is valued by all the professionals as important for success, in contrast to the aviation and medical world where surgeons and first pilots were more negative. This indicates that higher officers in the maritime domain, masters and alike are more positive to have their authority questioned compared to their counterparts in the aviation and health industry. In addition and irrelevant of position, this type of training seems to be perceived as something that they need to focus on.

However, even though level 1 and 2 of Kirkpatricks [22] taxonomy are the simplest forms of evaluation criteria, they serve an important purpose. Positive reactions and perceived learning are crucial in that they can provide evidence for credibility, to top level, within the industry and motivation for future learners. The opposite would have suggested a need to revise the "speak up" training programme. Thus we claim that these results are strong when professionals coping with the challenging Norwegian coastline on a daily basis report a highly relevant and educational course.

\section{CONCLUSIONS}

To sum up, we need to speak up within the maritime domain. The professional on board seems to acknowledge this call. Future research, however, must focus on change in behaviour on board towards better safety. The quest is to lower the incidents of near miss and accidents.

\section{ACKNOWLEDGEMENTS}

This project was sponsored, developed, certified and conducted by Royal Norwegian Naval Academy, and the Norwegian Express Coastal line. We would like to thank Cato Rasmussen (Cdr), Petter Lunde (Cdr) and Michel Hayes (Cdr) for thoughtful comments and perspectives on CRM training. Their contributions and professionalism as subject matter experts were essential for the project. A special thanks to the Norwegian Coastal Express, which saw the potential and organised time and resources to carry this through.

\section{REFERENCES}

1. Johnsen PE. Hurtigruta. Cappelens forlag. 1992.

2. Hansen HL. Surveillance of deaths on board Danish merchant ships, 1986-93: implications for prevention. Occup Environ Med. 1996; 53(4): 269-275, indexed in Pubmed: 8664966.

3. International Maritime Organization. Safer shipping demands safety culture. Paper presented at the World Maritime Day. 2002.

4. Rutherford JS, Flin R, Mitchell L. Non-technical skills of anaesthetic assistants in the perioperative period: a literature review. $\mathrm{Br} \mathrm{J} \mathrm{An}$ aesth. 2012; 109(1): 27-31, doi: 10.1093/bja/aes125, indexed in Pubmed: 22581808.

5. Salas E, Tannenbaum SI, Kraiger K, et al. The science of training and development in organizations: what matters in practice. Psychol Sci Public Interest. 2012; 13(2):
74-101, doi: 10.1177/1529100612436661, indexed in Pubmed: 26173283.

6. Salas E, Wilson KA, Burke CS, et al. Does crew resource management training work? An update, an extension, and some critical needs. Hum Factors. 2006; 48(2): 392-412, doi: 10.1518/001872006 777724444 , indexed in Pubmed: 16884057.

7. Musson DM, Helmreich RL. Team training and resource management in health care: current issues and future directions. Harvard Health Policy Review. 2004; 5(1): 25-35.

8. Lyndon A. Communication and teamwork in patient care: how much can we learn from aviation? J Obstet Gynecol Neonatal Nurs. 2006; 35(4): 538-546, doi: 10.1111/j.1552-6909.2006.00074.x, indexed in Pubmed: 16882000.

9. O'Connor P, Campbell J, Newon J, et al. Crew Resource Management Training Effectiveness: A Meta-Analysis and Some Critical Needs. Int J Aviat Psychol. 2008; 18(4): 353-368, doi: 10.1080/10508410802347044.

10. Beaty D. The naked pilot. Crowood. 2011.

11. Reid J, Bromiley M. Clinical human factors: the need to speak up to improve patient safety. Nurs Stand. 2012; 26(35): 35-40, doi: 10.7748/ns2012.05.26.35.35.c9084, indexed in Pubmed: 22708165.

12. Weick KE. The reduction of medical errors through mindful interdependence. In: Rosenthal MM, Sutchcliffe KM (Eds), Medical error: What do we know? What do we do? Jossey-Bass, San Francisco 2002: 177-199.

13. Ricci G, Pirillo I, Rinuncini C, et al. Medical assistance at the sea: legal and medico-legal problems. Int Marit Health. 2014; 65(4): 205-209, doi: 10.5603/IMH.2014.0039, indexed in Pubmed: 25522704.

14. Röttger S, Vetter S, Kowalski JT. Ship management attitudes and their relation to behavior and performance. Hum Factors. 2013; 55(3): 659-671, doi: 10.1177/0018720812461271, indexed in Pubmed: 23829038.

15. Canadian Transportation Safety Board. A safety study of operational relationship between masters/watchkeeping officers and marine pilots. 1995.

16. Burke CS, Salas E, Wilson-Donnelly K, et al. How to turn a team of experts into an expert medical team: guidance from the aviation and military communities. Qual Saf Health Care. 2004; 13 Suppl 1: i96-104, doi: 10.1136/qhc.13.suppl_1.i96, indexed in Pubmed: 15465963.

17. Gore DC, Powell JM, Baer JG, et al. Crew resource management improved perception of patient safety in the operating room. Am J Med Qual. 2009; 25(1): 60-63, doi: 10.1177/1062860609351236, indexed in Pubmed: 19966113.

18. Carney BT, West $P$, Neily JB, et al. Improving perceptions of teamwork climate with the Veterans Health Administration medical team training program. Am J Med Qual. 2011; 26(6): 480-484, doi: 10.1177/1062860611401653, indexed in Pubmed: 21813506.

19. Stout R, Cannon-Bowers J, Salas E, et al. Planning, Shared Mental Models, and Coordinated Performance: An Empirical Link Is Established. Human Factors. 1999; 41(1): 61-71, doi: 10.1518/0018 72099779577273.

20. Salas E, Cooke NJ, Rosen MA. On teams, teamwork, and team performance: discoveries and developments. Hum Factors. 2008; 50(3): 540-547, doi: 10.1518/001872008X288457, indexed in Pubmed: 18689065.

21. Salas E, DiazGranados D, Weaver SJ, et al. Does team training work? Principles for health care. Acad Emerg Med. 2008; 15(11): 
1002-1009, doi: 10.1111/j.1553-2712.2008.00254.x, indexed in Pubmed: 18828828.

22. Kirkpatrick DL. Evaluation of training. In: Craig RL (Ed.), Training and development handbook. A guide to human resources development. McGraw, New York, NY 1976: 18.1-18.27.

23. Eraut $\mathrm{M}$. Non-formal learning and tacit knowledge in professional work. Br J Educ Psychol. 2000; 70 (Pt 1): 113-136, indexed in Pubmed: 10765570.

24. Saus ER, Johnsen B, Eid J, et al. Who benefits from simulator training: Personality and heart rate variability in relation to situation awareness during navigation training. Computers in Human Behavior. 2012; 28(4): 1262-1268, doi: 10.1016/j.chb.2012.02.009.
25. Salas E, Sims DE, Burke CS. Is there a. Small Group Research. 2005; 36(5): 555-599, doi: 10.1177/1046496405277134.

26. Birzer M. The theory of andragogy applied to police training. Policing: An International Journal of Police Strategies \& Management. 2003; 26(1): 29-42, doi: 10.1108/13639510310460288.

27. Issenberg SB, McGaghie WC, Petrusa ER, et al. Features and uses of high-fidelity medical simulations that lead to effective learning: a BEME systematic review. Med Teach. 2005; 27(1): 10-28, doi: 10.1080/01421590500046924, indexed in Pubmed: 16147767.

28. Weller JM. Simulation in undergraduate medical education: bridging the gap between theory and practice. Med Educ. 2004; 38(1): 32-38, indexed in Pubmed: 14962024. 